\title{
Dynamics of the Stage Structured Population Model, Predator Accompanied by Michaelis Menten Holling Type Functional Responseand Delay and Prey Taking Refuge
}

\author{
N. Mohana Sorubha Sundari ${ }^{\mathrm{a}}$ and Dr. M. Valliathal ${ }^{\mathrm{b}}$ \\ a \\ Assistant Professor, Department of Mathematics, Chikkaiah Naicker College, \\ Erode-638 004. \\ ${ }^{\mathbf{b}}$ Assistant Professor, Department of Mathematics, Chikkaiah Naicker College, Erode-638 004.
}

Article History: Received: 11 January 2021; Accepted: 27 February 2021; Published online: 5 April 2021

\begin{abstract}
The current work considers predator prey system, prey taking refuge, predator reckoned with time delay and Michaelis Menten Holling type II response function undergoing two stages: juvenile and mature. From the characteristic equation, we derive conditions for the local stability of the system at the equilibrium points. Also, at the coexistence equilibrium point, the system is analyzed for the occurrence of Hopf bifurcation. Lyapunov function provides sufficient conditions for the global stability of the system. Numerical simulations are given to support the theory.
\end{abstract}

Keywords: Global stability,Michaelis Menten ,Stage structure,Prey refuge, Time delay

\section{Introduction}

An Ecosystem is composed of a group of species living in the same scenario. Interactions among several living organisms occur at different levels in the ecosystem which is vital for them to survive and thrive with their own species and these interactions play a major role in shaping the food web. One such interaction is between predator and prey. In fact, it is a complex system that involves many varieties of predator and preys and also involves multiple components like habitat, resource needs, individual and group behavior, population growth etc.,.Researchers, from Volterra (1920) attempted to study the qualitative features of such population dynamics by developing suitable mathematical models. Refinements of each model are under way by gradually integrating the factors (such as stage structure, refuge, time delay, harvest etc.) that affects the qualitative behaviors of the modelsand also by eluding the limitations in the developed models.

The functional response which is generally categorized as prey dependent, predator dependent and ratio dependent is one of the key components that determines the population model. Ratio dependent functional response is used when the predators search and share the food and also when the prey to predator abundance ratio defines the per capita growth rate of the predator. This model is studied extensively. It is well known that many species undergo different stages in their life span, especially immature and mature stage which in turn decides the size of their population at different periods of time. Therefore, stage structured mathematical model grabbed the scholars' attention and they started to research its impact on the dynamics of the system. Refuge is one of the predominant characters exhibited by the preys to avoid being food for the predators which is inevitable for them to survive and it also reduces the likelihood of mating that is reflected in their densities. Predator prey system can be better modeled using delay differential equations because it reveals many perplexing phenomena, making the model considered more rational.

Kar in 2006 studied the predator prey mechanism with Holling type II functional response by implementing prey refuge and harvest. Using harvesting efforts as control parameter, he arrived at conditions to hold the system in a required state [1]. Persistence theory on infinite dimensional system has been utilized in [2,3] to show that the delayed stage structure model is permanent if both prey and predator species coexists. Yang et al. in 2008 showed that the predator can survive even if the growth rate is negative for some period due to certain reasons in a periodic Holling type IV stage structured predator prey system [4].

Ratio dependent Holling Tanner model has been discussed by Liang and Pan in 2007. Through change of variables the model has been transformed into Lienard equation to obtain the uniqueness of limit cycle [5]. Saha and Chakrabarti [6] worked on the Holling Tanner delay model and proved that the system is permanent under certain conditions. By using blow up technique the qualitative behavior at $(0,0)$ has been explored. Classical ratio dependent model with Allee effect has been discussedand interesting phenomena such as cusp point, separatrix curve were observed [7]. Holling type II functional response coupled with modified Leslie Gower and prey refuge [8] has been studied with harvesting [9] and adequate conditions were obtained for the system to be stable both locally and globally. Applying Sotomayor's theorem, local bifurcation was studied along with Hopf bifurcation [10].

$\mathrm{Xu}$ and $\mathrm{Ma}$ in 2008 investigated ratio dependent predator prey model with gestation lag for the predator. Sufficient conditions for Hopf bifurcation and global stability (using comparison arguments) are obtained [11].Yu et al.introduced Michaelis Menten harvesting which is nonlinear in nature in a single species stage structured model and studied its qualitative behavior [12]. Prey, predator and top predator with functional response Michaelis Menten kind and two unequal delays is attempted by Dai et al. in 2014. They used normal form method and center manifold theorem to evaluate the properties of periodic solution [13]. Research have also been 
performed in Volterra type functional response, linear functional response, bilinear functional response [14-16] incorporated with stage structure and harvesting.

Mortoja et al. proposed a model with stage structure in both prey and predator with prey flaunting anti predator activity and group defense mechanism with different type of Holling type response function. Hopf bifurcation is analysed by considering transition rate as bifurcation parameter[17]. Using MATCONT software bifurcation and stability of a ratio-based model with noncontant predator harvesting rate were computed. Their analysis revealed that the system exhibits various form of bifurcation including bifurcations of Fold, Cusp and Bogdanov Takens [18].

Jost et al. in 1999 [19] studied the behavior of the predator prey system particularly at the origin with ratio dependent functional response.

$$
\begin{gathered}
\frac{d N}{d t}=R\left(1-\frac{N}{S}\right)-\frac{S N}{P+S N} P \\
\frac{d P}{d t}=\frac{S N}{P+S N} P-Q P
\end{gathered}
$$

Here $\mathrm{N}$ and $\mathrm{P}$ are prey abundance and predator abundance. They showed that the system exhibits different behaviors for different parameter values at the point $(0,0)$ and at this point the system has well defined dynamics. The above system has been consideredwith self-diffusion, cross diffusion and prey taking refuge for its spatial patterns [20].

$$
\begin{gathered}
\frac{\partial u}{\partial t}=d_{11} \Delta u+d_{12} \Delta v+u(1-u)-\frac{b u(1-M) v}{u(1-M)+v} \\
\frac{\partial v}{\partial t}=d_{21} \Delta u+d_{22} \Delta v-d v+\frac{e b u(1-M) v}{u(1-M)+v}
\end{gathered}
$$

Triggered by the research work of Xiao et al. [21], and keeping the basic model from Jost [19] and Sambath [20] we come up with a following population model. Here the predator is divided into two subgroups as juvenile and mature. Only the mature predator is capable of hunting and the immature predators depend solely on their elders for their food. Time delay due to gestation of mature predator and Michaelis Menten Holling type II functional response have been considered. The model takes the form

$$
\begin{aligned}
& \dot{u}(t)=R u(t)\left(1-\frac{u(t)}{K}\right)-\frac{A u(t)(1-\lambda) v_{2}(t)}{u(1-\lambda)+K_{1} v_{2}(t)} \\
& \dot{v}_{1}(t)=-D v_{1}(t)-d_{1} v_{1}(t)+\frac{B A(1-\lambda) u(t-\tau) v_{2}(t-\tau)}{u(t-\tau)(1-\lambda)+K_{1} v_{2}(t-\tau)}
\end{aligned}
$$

$$
\dot{v}_{2}(t)=D v_{1}(t)-d_{2} v_{2}(t)
$$

with initial conditions

$$
\begin{gathered}
\begin{array}{c}
u(\theta)=\phi_{1}(\theta), v_{1}(\theta)=\phi_{2}(\theta), v_{2}(\theta)=\phi_{3}(\theta) \\
\phi_{1}(\theta) \geq 0, \phi_{2}(\theta) \geq 0, \phi_{3}(\theta) \geq 0, \theta \in[-\tau, 0),
\end{array} \\
\phi_{1}(0)>0, \phi_{2}(0)>0, \phi_{3}(0)>0,
\end{gathered}
$$

where $\left(\phi_{1}(\theta), \phi_{2}(\theta), \phi_{3}(\theta)\right) \in C\left([-\tau, 0), \mathbb{R}_{+}^{3}\right)$, the continuous functions in Banach space mapping the interval $[-\tau, 0)$ into $\mathbb{R}_{+}^{3}=\left\{x_{1}, x_{2}, x_{3}: x_{i} \geq 0, i=1,2,3\right\}$.

Here $u(t), v_{1}(t), v_{2}(t)$ denotes size of prey, juvenile and adult predator respectively. $R, K, K_{1}$ represents the prey growth rate, environmental carrying capacity, predators benefit rate from cofeeding. $\lambda$ is the proportion of prey taking refuge and $\lambda \in[0,1) ; A, B, d_{1}, d_{2}$ are the capture rate, conversion coefficiency, death rate of immature and mature predators respectively. $\tau$ is the time lag due to gestation of mature predators.

This paper is sectioned as follows. In section 2 we show that the model system is bounded. We find equilibrium points and conditions for local stability at these equilibrium points. Hopf bifurcation at the positive equilibrium point is discussed in section 3. Permanence and global stability of the system at the coexistence equilibrium point are investigated in section 4. Numerical simulations are given in section 5. Section 6 is the conclusion. 


\section{POSITIVITY, BOUNDEDNESS, EQUILIBRIA AND LOCAL STABILITY}

\subsection{Positivity}

The proposed system (1-3) can be written in the matrix form as $\dot{X}=P(X)$

where $X=\left(u, v_{1}, v_{2}\right)^{T}$ and

$$
P(X)=\left[\begin{array}{c}
P_{1}(X) \\
P_{2}(X) \\
P_{3}(X)
\end{array}\right]=\left[\begin{array}{c}
R u(t)\left(1-\frac{u(t)}{K}\right)-\frac{A u(t)(1-\lambda) v_{2}(t)}{u(1-\lambda)+K_{1} v_{2}(t)} \\
-D v_{1}(t)-d_{1} v_{1}(t)+\frac{B A(1-\lambda) u(t-\tau) v_{2}(t-\tau)}{u(t-\tau)(1-\lambda)+K_{1} v_{2}(t-\tau)} \\
D v_{1}(t)-d_{2} v_{2}(t)
\end{array}\right]
$$

It can be easily verified that $P_{i}(X) /_{X_{i=0}} \geq 0, \mathrm{X} \in \mathbb{R}_{+}^{3}$ for $\mathrm{i}=1,2,3$. Due to Nagumo's theorem any solution of (1-3) with initial conditions $X(0)=X_{0} \in \mathbb{R}_{+}^{3}$ always lies in $\mathbb{R}_{+}^{3}$ for all $t \geq 0$. (ie) it remains positive throughout the region for all finite time.

\subsection{Boundedness}

Theorem 2.1: All the solutions of the system (1-3) with initial conditions (4) are uniformly bounded for all $t \geq 0$.

Proof: Let

$\mathbb{V}(t)=B u(t-\tau)+v_{1}(t)+v_{2}(t)$

Taking the time derivative of the above along the nonnegative solution of (1-3),

$$
\begin{gathered}
\dot{\mathbb{V}}(t)=B \dot{u}(t-\tau)+\dot{v}_{1}(t)+\dot{v}_{2}(t) \\
\dot{\mathbb{V}}(t)=B R u(t-\tau)\left[1-\frac{u(t-\tau)}{K}\right]-\frac{A(1-\lambda) u(t-\tau) v_{2}(t-\tau)}{u(t-\tau)(1-\lambda)+K_{1} v_{2}(t-\tau)}-D v_{1}(t)-d_{1} v_{1}(t) \\
+\frac{B A(1-\lambda) u(t-\tau) v_{2}(t-\tau)}{u(t-\tau)(1-\lambda)+K_{1} v_{2}(t-\tau)}+D v_{1}(t)-d_{2} v_{2}(t) \\
=B R u(t-\tau)\left[1-\frac{u(t-\tau)}{K}\right]-d_{1} v_{1}(t)-d_{2} v_{2}(t)
\end{gathered}
$$

Let $S=\min \left\{d_{1}, d_{2}\right\}$

$$
\begin{aligned}
& \dot{\mathbb{V}}(t)+S \mathbb{V}(t)=B R u(t-\tau)\left[1-\frac{u(t-\tau)}{K}\right]-d_{1} v_{1}(t)-d_{2} v_{2}(t)+B S u(t-\tau)+S v_{1}(t)+S v_{2}(t) \\
& =B u(t-\tau)\left[R\left[1-\frac{u(t-\tau)}{K}\right]+S\right]-\left(S-d_{1}\right) v_{1}(t)-\left(S-d_{2}\right) v_{2}(t) \\
& \leq B u(t-\tau)\left[R\left[1-\frac{u(t-\tau)}{K}\right]+S\right] \\
& \dot{\mathbb{V}}(t)+S \mathbb{V}(t) \leq M \text { where } M=\frac{B K(R+S)^{2}}{4} \\
& \therefore \mathbb{V}(t) \leq \frac{M}{S}+\left[v(0)-\frac{M}{S}\right] e^{-s t} \\
& \lim _{t \rightarrow \infty} \mathbb{V}(t) \leq \frac{M}{S} \forall t>0
\end{aligned}
$$

Hence all the solutions of the considered system are uniformly bounded. Hence the theorem.

\subsection{Equilibria}

Solving the system of equations (1-3) we obtain the trivial equilibrium point $E_{0}(0,0,0)$, predator free equilibrium $E_{1}(K, 0,0)$ and $E_{c}\left(u^{c}, v_{1}^{c}, v_{2}^{c}\right)$.The coexistence equilibrium $E_{c}\left(u^{c}, v_{1}^{c}, v_{2}^{c}\right)$ exists if the following conditions hold 
$\left(\mathrm{C}_{1}\right)$

$$
B A-r_{1}>0, K_{1} R B>(1-\lambda)\left(B A-r_{1}\right)
$$

where

$$
u^{c}=K-\frac{K(1-\lambda)\left(B A-r_{1}\right)}{K_{1} R B} ; v_{1}^{c}=\frac{d_{2}}{D} v_{2}^{c} ; v_{2}^{c}=\frac{u^{c}(1-\lambda)\left(B A-r_{1}\right)}{K_{1} r_{1}}
$$

where

$$
r_{1}=d_{2}\left(1+\frac{d_{1}}{D}\right)
$$

If $\hat{E}=\left(\hat{u}, \hat{v}_{1}, \hat{v}_{2}\right)$ be any arbitrary equilibrium point, then the variational matrix of the system $(1-3)$ at this point is given by

$J=\left[\begin{array}{ccc}R-\frac{2 R \widehat{u}}{K}-\frac{A(1-\lambda) K_{1} \hat{v}_{2}^{2}}{\left[\widehat{u}(1-\lambda)+K_{1} \hat{v}_{2}\right]^{2}} & 0 & -\frac{A \widehat{u}^{2}(1-\lambda)^{2}}{\left[\widehat{u}(1-\lambda)+K_{1} \hat{v}_{2}\right]^{2}} \\ \frac{K_{1} B A(1-\lambda) \hat{v}_{2}^{2} e^{-\lambda \tau}}{\left[\widehat{u}(1-\lambda)+K_{1} \hat{v}_{2}\right]^{2}} & -D-d_{1} & \frac{B A(1-\lambda)^{2} \widehat{u}^{2} e^{-\lambda \tau}}{\left[\widehat{u}(1-\lambda)+K_{1} \hat{v}_{2}\right]^{2}} \\ 0 & D & -d_{2}\end{array}\right]$

\subsection{Local Stability}

Theorem 2.2: The zero equilibrium $E_{0}(0,0,0)$ is unstable for the system (1-3).

Proof: Working out the characteristic equation from the variational matrix and substituting the equilibrium point $E_{0}(0,0,0)$ we get,

$(\gamma-R)\left(\gamma+D+d_{1}\right)\left(\gamma+d_{2}\right)=0$

We observe that one of the roots is positive in (9) and hence the equilibrium $E_{0}$ is always unstable. Hence the theorem.

Theorem 2.3: The predator free equilibrium $E_{1}(K, 0,0)$ of the system (1-3) is locally asymptotically stable if the following holds

$\left(B A-r_{1}\right)<0\left(\mathrm{C}_{2}\right)$

Proof: At the equilibrium point $E_{1}(K, 0,0)$, the characteristic equation of (8) is

$(\gamma+R)\left[\left(\gamma+D+d_{1}\right)\left(\gamma+d_{2}\right)-A B D e^{-\gamma \tau}\right]=0$

Clearly from (10) one root is negative. Therefore, the remaining roots are determined by

$\gamma^{2}+\left(D+d_{1}+d_{2}\right) \gamma+\left(D+d_{1}\right) d_{2}-A B D e^{-\gamma \tau}=0$

When $\tau=0$, (11) becomes

$\gamma^{2}+\left(D+d_{1}+d_{2}\right) \gamma+\left(D+d_{1}\right) d_{2}-A B D=0$

Using Routh Hurwitz criterion, the boundary equilibrium $E_{1}(K, 0,0)$ is locally asymptotically stable for $\left(\mathrm{C}_{2}\right)$.

The existence of purely imaginary root of (11) is analyzed for $\tau>0$. Let $i \Omega_{1}$ where $\Omega_{1}>0$ be the root of (11). Then,

$$
-\Omega_{1}^{2}+\left(D+d_{1}+d_{2}\right) i \Omega_{1}+\left(D+d_{1}\right) d_{2}-A B D \cos \Omega_{1} \tau+i A B D \sin \Omega_{1} \tau=0
$$

Separating the real and imaginary part,$$
-\Omega_{1}^{2}+\left(D+d_{1}\right) d_{2}=A B D \cos \Omega_{1} \tau
$$ 
$\left(D+d_{1}+d_{2}\right) \Omega_{1}=A B D \sin \Omega_{1} \tau$

which gives

$\Omega_{1}^{4}+\left[\left(D+d_{1}\right)^{2}+d_{2}{ }^{2}\right] \Omega_{1}^{2}+\left(D+d_{1}\right)^{2} d_{2}{ }^{2}-A^{2} B^{2} D^{2}=0$

If the condition $\left(\mathrm{C}_{2}\right)$ is satisfied, then all the eigen values of (11) have negative real parts for all $\tau \geq 0$, which in turn shows that the boundary equilibrium is locally asymptotically stable.(by theorem 3.4.1 [22]). Hence the theorem.

Now, let us investigate the stability of the equilibriumpoint $E_{c}$. The characteristic equation at the coexistence equilibrium point is

$\gamma^{3}+h_{2} \gamma^{2}+h_{1} \gamma+h_{0}+\left(g_{1} \gamma+g_{0}\right) e^{-\gamma \tau}=0$

where

$$
\begin{gathered}
h_{2}=D+d_{1}+d_{2}+\mathcal{A}_{1}+\frac{2 R u^{c}}{K}-R \\
h_{1}=Q_{1}+\left[\mathcal{A}_{1}+\frac{2 R u^{c}}{K}-R\right]\left[D+d_{1}+d_{2}\right] \\
h_{0}=Q_{1}\left[\mathcal{A}_{1}+\frac{2 R u^{c}}{K}-R\right]
\end{gathered}
$$

$g_{1}=-\mathcal{B}_{1}$

$$
g_{0}=-\mathcal{B}_{1}\left[\frac{2 R u^{c}}{K}-R\right]
$$

and

$\mathcal{A}_{1}=\frac{A(1-\lambda) K_{1} v_{2}^{c^{2}}}{\left[u^{c}(1-\lambda)+K_{1} v_{2}^{c}\right]^{2}} ; \mathcal{B}_{1}=\frac{A B D(1-\lambda)^{2} u^{c^{2}}}{\left[u^{c}(1-\lambda)+K_{1} v_{2}^{c}\right]^{2}}=\frac{Q_{1} d_{2}\left(1+\frac{d_{1}}{D}\right)}{A B} ; Q_{1}=\left(D+d_{1}\right) d_{2}$

when $\tau=0$, (16) becomes

$$
\gamma^{3}+h_{2} \gamma^{2}+\left(h_{1}+g_{1}\right) \gamma+h_{0}+g_{0}=0
$$

From $\left(\mathrm{C}_{1}\right)$, we obtain $\mathcal{B}_{1}<Q_{1}$

where

$$
\begin{gathered}
h_{2}=D+d_{1}+d_{2}+\mathcal{A}_{1}+\frac{2 R u^{c}}{K}-R \\
h_{1}+g_{1}=\left[Q_{1}-\mathcal{B}_{1}\right]+\left[\mathcal{A}_{1}+\frac{2 R u^{c}}{K}-R\right]\left[D+d_{1}+d_{2}\right] \\
h_{0}+g_{0}=Q_{1} \mathcal{A}_{1}+\left[\mathcal{Q}_{1}-\mathcal{B}_{1}\right] \frac{2 R u^{c}}{K}-\left[Q_{1}-\mathcal{B}_{1}\right] R \\
h_{2}\left(h_{1}+g_{1}\right)-\left(h_{0}+g_{0}\right)=\left[D+d_{1}+d_{2}+\mathcal{A}_{1}+\frac{2 R u^{c}}{K}-R\right]\left[\mathcal{Q}_{1}-\mathcal{B}_{1}\right]+\left[\mathcal{A}_{1}+\frac{2 R u^{c}}{K}-R\right] \\
+\left[\mathcal{A}_{1}+\frac{2 R u^{c}}{K}-R\right]\left[\left(D+d_{1}\right)\left(D+d_{1}+\mathcal{A}_{1}+\frac{2 R u^{c}}{K}-R\right)\right]+d_{2}\left[\mathcal{A}_{1}+\frac{2 R u^{c}}{K}-R+d_{2}\right]
\end{gathered}
$$


Denote

$$
+Q_{1} \mathcal{A}_{1}+\left[Q_{1}+\mathcal{B}_{1}\right] \frac{2 R u^{c}}{K}-\left[Q_{1}+\mathcal{B}_{1}\right] R
$$

$$
\begin{gathered}
P_{1}=\frac{Q_{1} \mathcal{A}_{1}}{\left[Q_{1}+\mathcal{B}_{1}\right]}+\frac{2 R u^{c}}{K} ; \mathrm{P}_{2}=\mathcal{A}_{1}+\frac{2 R u^{c}}{K} ; \mathrm{P}_{3}=\frac{Q_{1} \mathcal{A}_{1}}{\left[Q_{1}-\mathcal{B}_{1}\right]}+\frac{2 R u^{c}}{K} \\
P_{4}=\frac{\left[D+d_{1}+d_{2}+P_{2}-R\right]\left[Q_{1}-\mathcal{B}_{1}\right]}{\left[Q_{1}+\mathcal{B}_{1}\right]}+\frac{\left[P_{2}-R\right]\left[\left(D+d_{1}\right)\left(D+d_{1}+P_{2}-R\right)+d_{2}\left(P_{2}-R+d_{2}\right)\right]}{\left[Q_{1}+\mathcal{B}_{1}\right]}+P_{1}
\end{gathered}
$$

If $R<\min \left\{P_{2}, P_{4}\right\}$ and $\left(\mathrm{C}_{1}\right)$ hold then $h_{2}>0,\left(h_{1}+g_{1}\right)>0,\left(h_{0}+g_{0}\right)>0$ and

$h_{2}\left(h_{1}+g_{1}\right)-\left(h_{0}+g_{0}\right)>0$.Utilizing the criterion given by Hurwitz, the system (1-3) is locally asymptotically stable at the positive equilibrium $E_{c}\left(u^{c}, v_{1}^{c}, v_{2}^{c}\right)$.

For $\tau>0, i \Omega$ where $\Omega>0$ is a solution of (16) if and only if it satisfies

$$
-i \Omega^{3}-h_{2} \Omega^{2}+i h_{1} \Omega+h_{0}+i g_{1} \Omega \cos \Omega \tau+g_{1} \Omega \sin \Omega \tau+g_{0} \cos \Omega \tau-i g_{0} \sin \Omega \tau=0
$$

Separating the real and imaginary part,

$$
\begin{aligned}
& \Omega^{3}-h_{1} \Omega=g_{1} \Omega \cos \Omega \tau-g_{0} \sin \Omega \tau \\
& h_{2} \Omega^{2}-h_{0}=g_{1} \Omega \sin \Omega \tau+g_{0} \cos \Omega \tau
\end{aligned}
$$

which gives,

$$
\Omega^{6}+\left(h_{2}^{2}-2 h_{1}\right) \Omega^{4}+\left(h_{1}^{2}-2 h_{2} h_{0}-g_{1}^{2}\right) \Omega^{2}+h_{0}^{2}-g_{0}^{2}=0
$$

where

$$
\begin{aligned}
& \left(h_{2}^{2}-2 h_{1}\right)=\left(D+d_{1}\right)^{2}+d_{2}^{2}+\left(\mathcal{A}_{1}+\frac{2 R u^{c}}{K}-R\right)^{2}>0 \\
& h_{1}^{2}-2 h_{2} h_{0}-g_{1}^{2}=\left[Q_{1}+\left(\mathcal{A}_{1}+\frac{2 R u^{c}}{K}-R\right)\left(D+d_{1}+d_{2}\right)\right]^{2} \\
& -2\left[D+d_{1}+d_{2}+\mathcal{A}_{1}+\frac{2 R u^{c}}{K}-R\right]\left[\mathcal{Q}_{1}\left(\mathcal{A}_{1}+\frac{2 R u^{c}}{K}-R\right)\right]-\mathcal{B}_{1}^{2} \\
& =\left[\mathcal{A}_{1}+\frac{2 R u^{c}}{K}-R\right]^{2}\left[\left(D+d_{1}\right)^{2}+d_{2}^{2}\right]>0 \\
& h_{0}^{2}-g_{0}^{2}=Q_{1}^{2}\left[\mathcal{A}_{1}+\frac{2 R u^{c}}{K}-R\right]^{2}-\mathcal{B}_{1}^{2}\left[\frac{2 R u^{c}}{K}-R\right]^{2} \\
& =\left[Q_{1} \mathcal{A}_{1}+\left(Q_{1}+\mathcal{B}_{1}\right) \frac{2 R u^{c}}{K}-\left(Q_{1}+\mathcal{B}_{1}\right) R\right]\left[Q_{1} \mathcal{A}_{1}+\left(Q_{1}-\mathcal{B}_{1}\right) \frac{2 R u^{c}}{K}-\left(Q_{1}-\mathcal{B}_{1}\right) R\right]
\end{aligned}
$$

If $R<P_{1}$ and $\left(\mathrm{C}_{1}\right)$ holds then, $h_{0}^{2}-g_{0}^{2}>0$ which shows that (20) has no positive roots. Therefore by a theorem [22], for all $\tau \geq 0$, all the eigen values of (20) have negative real parts. Hence at the positive equilibrium $E_{c}\left(u^{c}, v_{1}^{c}, v_{2}^{c}\right)$ the system is locally asymptotically stable for all $\tau \geq 0$.

If $P_{1}<R<\min \left\{P_{2}, P_{4}\right\}$, then $h_{0}^{2}-g_{0}^{2}<0$. Then there exists a unique positive root $\Omega_{*}$ which satisfies (20). From (18) and (19) we have,

$$
g_{1} \Omega_{*}^{4}+\left(h_{2} g_{0}-h_{1} g_{1}\right) \Omega_{*}^{2}-h_{0} g_{0}=\left(g_{1}^{2} \Omega_{*}^{2}+g_{0}^{2}\right) \cos \Omega_{*} \tau
$$

$\cos \Omega_{*} \tau=\frac{g_{1} \Omega_{*}^{4}+\left(h_{2} g_{0}-h_{1} g_{1}\right) \Omega_{*}^{2}-h_{0} g_{0}}{\left(g_{1}^{2} \Omega_{*}^{2}+g_{0}^{2}\right)}$ 
Let

$\tau_{* n}=\frac{1}{\Omega_{*}} \arccos \frac{g_{1} \Omega_{*}^{4}+\left(h_{2} g_{0}-h_{1} g_{1}\right) \Omega_{*}^{2}-h_{0} g_{0}}{\left(g_{1}^{2} \Omega_{*}^{2}+g_{0}^{2}\right)}+\frac{2 n \pi}{\Omega_{*}}, n=0,1,2, \ldots$

If $h_{0}^{2}-g_{0}^{2}<0$, then $E_{c}$ remains stable for $\tau<\tau_{0}:=\tau_{* 0}$.

Now we derive that $\left\{\frac{d(\operatorname{Re} \gamma)}{d \tau}\right\}_{\tau=\tau_{0}}>0$. Differentiating (16) with respect to $\tau$, it follows that

$\left[\frac{d \gamma}{d \tau}\right]^{-1}=-\frac{3 \gamma^{2}+2 h_{2} \gamma+h_{1}}{\gamma\left(\gamma^{3}+h_{2} \gamma^{2}+h_{1} \gamma+h_{0}\right)}+\frac{g_{1}}{\gamma\left(g_{1} \gamma+g_{0}\right)}-\frac{\tau}{\gamma}$

Simplifying,

$$
\begin{aligned}
\operatorname{sgn}\left\{\frac{d(\operatorname{Re} \gamma)}{d \tau}\right\}_{\tau=\Omega_{*}} & =\operatorname{sgn}\left\{\operatorname{Re}\left[\frac{d \gamma}{d \tau}\right]^{-1}\right\} \\
& =\sin \left\{-\frac{\left[h_{1}-3 \Omega_{*}^{2}\right]\left[\Omega_{*}^{2}-h_{1}\right]+2 h_{2}\left[h_{0}-h_{2} \Omega_{*}^{2}\right]}{\left[\Omega_{*}^{3}-h_{1} \Omega_{*}\right]^{2}+\left[h_{0}-h_{2} \Omega_{*}^{2}\right]^{2}}-\frac{\left[g_{1}^{2}\right]}{\left[g_{1}^{2} \Omega_{*}^{2}+g_{0}^{2}\right]}\right\}
\end{aligned}
$$

From (18) and (19) we get,

$$
\left[\Omega_{*}^{3}-h_{1} \Omega_{*}\right]^{2}+\left[h_{0}-h_{0} \Omega_{*}^{2}\right]^{2}=\left[g_{1}^{2} \Omega_{*}^{2}+g_{0}^{2}\right]
$$

Therefore

$\sin \left\{\frac{d(\operatorname{Re} \gamma)}{d \tau}\right\}=\sin \left\{\frac{3 \Omega_{*}^{4}+2\left(h_{2}^{2}-2 h_{1}\right) \Omega_{*}^{2}+h_{1}^{2}-2 h_{0} h_{2}-g_{1}^{2}}{\left[g_{1}^{2} \Omega_{*}^{2}+g_{0}^{2}\right]}\right\}>0$

Thus, the traversal condition holds and Hopf bifurcation occurs at $\Omega=\Omega_{*}, \tau=\tau_{0}$. Summarizing the above we get

Theorem: 2.4(1) If $R<\min \left\{P_{1}, P_{2}, P_{4}\right\}$ and $\left(\mathrm{C}_{1}\right)$ hold, then the positive equilibrium $E_{c}$ of the system $(1-3)$ is locally asymptotically stable for all $\geq 0$.

(2) If $P_{1}<R<\min \left\{P_{2}, P_{4}\right\}$ and $\left(\mathrm{C}_{1}\right)$ holds, then there exists a $\tau_{0}>0$ such that when $\tau \epsilon\left[0, \tau_{0}\right)$, the positive equilibrium $E_{c}$ is locally asymptotically stable. Also, the system (1-3) undergoes a Hopf bifurcation at $E_{c}=$ $\left(u^{c}, v_{1}^{c}, v_{2}^{c}\right)$ when $=\tau_{0}$.

\section{Permanence}

Lemma 3.1: Let $\left(u(t), v_{1}(t), v_{2}(t)\right)$ be any positive solution of the system (1-3) with initial conditions (4). Assume $\left(\mathrm{C}_{1}\right)$ holds then,

$\lim _{t \rightarrow \infty} \sup u(t) \leq L_{1}, \lim _{t \rightarrow \infty} \sup v_{1}(t) \leq L_{2}, \lim _{t \rightarrow \infty} \sup v_{2}(t) \leq L_{3}$

where

$$
L_{1}=K, L_{2}=\frac{(1-\lambda) B A D L_{1}-(1-\lambda) d_{2}\left(D+d_{1}\right) L_{1}}{D\left(D+d_{1}\right) K_{1}}, L_{3}=\frac{D}{d_{2}} L_{2}
$$

Proof: Let $\left(u(t), v_{1}(t), v_{2}(t)\right)$ be any positive solution of the system (1-3) with initial conditions (4). From (1) we get,

$$
\dot{u}(t)=R u(t)\left(1-\frac{u(t)}{k}\right)-\frac{A u(t)(1-\lambda) v_{2}(t)}{u(t)(1-\lambda)+K_{1} v_{2}(t)}
$$

$$
\leq R u(t)\left(1-\frac{u(t)}{k}\right)
$$

Using lemma (2.3) in [23] to the above, it immediately follows that

$$
\lim _{t \rightarrow \infty} \sup u(t) \leq K:=L_{1}(23)
$$


Then for $>0$, sufficiently small, there exists a $T_{1}>0$, such that if $t>T_{1}, u(t) \leq L_{1}+\varepsilon$. From equations (2) and (3), we get for $t>T_{1}+\tau$,

$$
\begin{gathered}
\dot{v}_{1}(t)=-\left(D+d_{1}\right) v_{1}(t)+\frac{B A(1-\lambda)\left(L_{1}+\varepsilon\right) v_{2}(t-\tau)}{\left(L_{1}+\varepsilon\right)(1-\lambda)+K_{1} v_{2}(t-\tau)} \\
\dot{v}_{2}(t)=D v_{1}(t)-d_{2} v_{2}(t)(24)
\end{gathered}
$$

Consider the following auxiliary equations

$$
\begin{gathered}
\dot{u}_{1}(t)=-\left(D+d_{1}\right) u_{1}(t)+\frac{B A(1-\lambda)\left(L_{1}+\varepsilon\right) u_{2}(t-\tau)}{\left(L_{1}+\varepsilon\right)(1-\lambda)+K_{1} u_{2}(t-\tau)} \\
\dot{u}_{2}(t)=D u_{1}(t)-d_{2} u_{2}(t)(25)
\end{gathered}
$$

Following the proof of Lemma 2.4 in [23] we get,

$\lim _{t \rightarrow \infty} u_{1}(t)=\frac{D B A(1-\lambda)\left(L_{1}+\varepsilon\right)-\left(D+d_{1}\right) d_{2}\left(L_{1}+\varepsilon\right)(1-\lambda)}{\left(D+d_{1}\right) K_{1} D}:=L_{2 \varepsilon}$

$\lim _{t \rightarrow \infty} u_{2}(t)=\frac{D}{d_{2}} L_{2 \varepsilon}:=L_{3 \varepsilon}$

By comparison we obtain,

$\lim _{t \rightarrow \infty} \sup _{1}(t) \leq L_{2 \varepsilon}, \lim _{t \rightarrow \infty} \sup u_{2}(t) \leq L_{3 \varepsilon}(27)$

Let $\rightarrow 0$, it follows that

$\lim _{t \rightarrow \infty} \sup v_{1}(t) \leq L_{2}, \lim _{t \rightarrow \infty} \sup v_{2}(t) \leq L_{3}(28)$

Hence the proof.

Note: It follows from $\left(\mathrm{C}_{1}\right)$ that $L_{2}=\frac{D B A(1-\lambda) L_{1}-\left(D+d_{1}\right) d_{2} L_{1}(1-\lambda)}{\left(D+d_{1}\right) K_{1} D}>0$. Hence there exists a positive constant $\varepsilon$ such that $L_{2 \varepsilon}>0, L_{3 \varepsilon}>0$.

Lemma 3.2: Let $\left(u(t), v_{1}(t), v_{2}(t)\right)$ be any positive solution of the system (1-3) with initial conditions (4). If $\left(\mathrm{C}_{1}\right)$ and

$$
R>\frac{A(1-\lambda)}{K_{1}} \text { holds, then }
$$

$\lim _{t \rightarrow \infty} \inf u(t) \geq l_{1}, \lim _{t \rightarrow \infty} \inf v_{1}(t) \geq l_{2}, \lim _{t \rightarrow \infty} \inf v_{2}(t) \geq l_{3}(30)$

where $l_{1}=\frac{K\left[K_{1} R-A(1-\lambda)\right]}{R K_{1}}, l_{2}=\frac{(1-\lambda) B A D l_{1}-(1-\lambda) d_{2}\left(D+d_{1}\right) l_{1}}{D\left(D+d_{1}\right) K_{1}}, \quad l_{3}=\frac{D}{d_{2}} l_{2}$

Proof: It follows from $\left(\mathrm{C}_{1}\right)$ and condition (29) that

$$
\begin{gathered}
l_{1}=\frac{K\left[K_{1} R-A(1-\lambda)\right]}{R K_{1}}>0, \\
l_{2}=\frac{(1-\lambda) B A D l_{1}-(1-\lambda) d_{2}\left(D+d_{1}\right) l_{1}}{D\left(D+d_{1}\right) K_{1}}>0
\end{gathered}
$$

Hence, there exists enough positive constants $\varepsilon$ sufficiently small such that

$$
l_{1 \varepsilon}=\frac{K\left[K_{1} R-A(1-\lambda)\right]}{R K_{1}}>0
$$




$$
\begin{gathered}
l_{2 \varepsilon}=\frac{(1-\lambda) B A D\left(l_{1}-\varepsilon\right)-(1-\lambda) d_{2}\left(D+d_{1}\right)\left(l_{1}-\varepsilon\right)}{D\left(D+d_{1}\right) K_{1}}>0 \\
l_{3 \varepsilon}=\frac{D}{d_{2}} l_{2 \varepsilon}>0
\end{gathered}
$$

Let $\left(u(t), v_{1}(t), v_{2}(t)\right)$ be any positive solution of the system (1-3) with initial conditions (4). For the above $\varepsilon>0$, sufficiently small, it follows from the previous lemma that there exists a $T_{2}>$ 0 such that if $t>T_{2}, v_{1}(t) \leq L_{2}+\varepsilon, v_{2}(t) \leq L_{3}+\varepsilon$. Hence for $t>T_{2}$, we have from equation (1)

$$
\dot{u}(t) \geq u(t)\left[R-\frac{R}{K} u(t)-\frac{A(1-\lambda)}{K_{1}}\right]
$$

Using condition (29) we have,

$\lim _{x \rightarrow \infty} \inf u(t) \geq \frac{\left[K_{1} R-A(1-\lambda)\right] K}{R K_{1}}:=l_{1}>0$

For the above $\varepsilon>0$ sufficiently small, there exists a $T_{3} \geq T_{2}, u(t) \geq l_{1}-\varepsilon$. Therefore from equations(2) and (3), for $t>T_{3}+\tau$

$$
\dot{v}_{1}(t)=-\left(D+d_{1}\right) v_{1}(t)+\frac{B A(1-\lambda)\left(l_{1}-\varepsilon\right) v_{2}(t-\tau)}{\left(l_{1}-\varepsilon\right)(1-\lambda)+K_{1} v_{2}(t-\tau)}
$$

$\dot{v}_{2}(t)=D v_{1}(t)-d_{2} v_{2}(t)$

Consider the following auxiliary equations

$$
\dot{u}_{1}(t)=-\left(D+d_{1}\right) u_{1}(t)+\frac{B A(1-\lambda)\left(l_{1}-\varepsilon\right) u_{2}(t-\tau)}{\left(l_{1}-\varepsilon\right)(1-\lambda)+K_{1} u_{2}(t-\tau)}
$$

$\dot{u}_{2}(t)=D u_{1}(t)-d_{2} u_{2}(t)$

Following the proof of the lemma 2.4 in [23], we obtain that

$\lim _{t \rightarrow \infty} u_{1}(t) \geq l_{2 \varepsilon} ; \lim _{t \rightarrow \infty} u_{2}(t) \geq l_{3 \varepsilon}$

By comparison we get,

$$
\begin{gathered}
\lim _{t \rightarrow \infty} \inf v_{1}(t) \geq l_{2 \varepsilon}, \lim _{t \rightarrow \infty} \inf v_{2}(t) \geq l_{3 \varepsilon} \\
\text { Let } \varepsilon \rightarrow 0, \text { then } \\
\lim _{t \rightarrow \infty} \inf v_{1}(t) \geq l_{2}, \lim _{t \rightarrow \infty} \inf v_{2}(t) \geq l_{3}
\end{gathered}
$$

The proof is complete.

Theorem 3.1: Assume that $\left(C_{1}\right)$ and (29) hold. Then the system (1-3) is permanent.

Proof: It follows from lemma (3.1) and (3.2).

\section{Global Stability}

Here we use Lyapunov functional and Laselle invariance principal to study the global attractivity of the coexistence equilibrium $E_{c}$ of the system.

Theorem 4.1: Assume that $\left(C_{1}\right)$ and (29) are satisfied. If

$$
\frac{R}{K}\left(l_{1}+u^{c}\right)-R \geq \bar{\rho}_{1}, \mathrm{u}^{c^{2}}(1-\lambda) \geq \bar{\rho}_{2}
$$

where 


$$
\begin{aligned}
& \bar{\rho}_{1}=\frac{\mathrm{AK}_{1}\left[L_{1}^{2} u^{c} v_{2}^{c}(1-\lambda)+u^{\left.c^{2} L_{3} K_{1} v_{2}^{c}+2 L_{1} v_{2}^{c^{2}} K_{1} L_{3}\right]}\right.}{2 l_{1} u^{c}\left[l_{1}(1-\lambda)+K_{1} l_{3}\right]\left[u^{c}(1-\lambda)+K_{1} v_{2}^{c}\right]}, \\
& \bar{\rho}_{2}=\frac{\left[L_{1}^{2}(1-\lambda)+u^{c} L_{3} K_{1}\right]}{2 l_{1}}
\end{aligned}
$$

then at the positive equilibrium $E_{c}\left(u^{c}, v_{1}^{c}, v_{1}^{c}\right)$ the system (1-3) is globally stable.

Proof: Let $\left(u(t), v_{1}(t), v_{2}(t)\right)$ be any positive solution of the system (1-3) with initial conditions (4). Define

$$
V(t)=u-u^{c}-u^{c} \log \frac{u}{u^{c}}+C_{1}\left[v_{1}-v_{1}^{c}-v_{1}^{c} \log \frac{v_{1}}{v_{1}^{c}}\right]+C_{2}\left[v_{2}-v_{2}^{c}-v_{2}^{c} \log \frac{v_{2}}{v_{2}^{c}}\right]
$$

Taking the time derivative of $V(t)$ along the positive solution of the system (1-3), we get

$$
\begin{aligned}
& \dot{V}(t)=\left(1-\frac{u^{c}}{u}\right)\left[R u\left(1-\frac{u}{K}\right)-\frac{A u(1-\lambda) v_{2}}{u(1-\lambda)+K_{1} v_{2}}\right]+C_{1}\left(1-\frac{v_{1}^{c}}{v_{1}}\right)\left[-\left(D+d_{1}\right) v_{1}\right. \\
& \left.+\frac{B A(1-\lambda) u(t-\tau) v_{2}(t-\tau)}{u(t-\tau)(1-\lambda)+K_{1} v_{2}(t-\tau)}\right] \\
& +\mathrm{C}_{2}\left(1-\frac{v_{2}^{c}}{v_{2}}\right)\left[D v_{1}-d_{2} v_{2}\right] \\
& =\frac{\left(u-u^{c}\right)^{2}}{u}\left[R-\frac{R}{K}\left(u+u^{c}\right)\right]+\left[1-\frac{u^{c}}{u}\right] \frac{A u^{c}(1-\lambda) v_{2}^{c}}{u^{c}(1-\lambda)+K_{1} v_{2}^{c}}-A u(1 \\
& -\lambda) v_{2}\left[\frac{u^{c}(1-\lambda)+K_{1} v_{2}^{c}}{\left[u(1-\lambda)+K_{1} v_{2}\right] u^{c}(1-\lambda)}-\frac{u^{c}}{u^{2}(1-\lambda)}\right] \\
& +\frac{K_{1}(1-\lambda)\left[v_{2}^{c} u^{2}-v_{2} u^{c^{2}}\right]}{u^{c}(1-\lambda)\left[u(1-\lambda)+K_{1} v_{2}\right]} \frac{A v_{2}}{u}+C_{1}\left(D+d_{1}\right) v_{1}^{c}+\frac{C_{1} B A(1-\lambda) u(t-\tau) v_{2}(t-\tau)}{u(t-\tau)(1-\lambda)+K_{1} v_{2}(t-\tau)} \\
& -\frac{C_{1} v_{1}^{c} B A(1-\lambda) u(t-\tau) v_{2}(t-\tau)}{v_{1}\left[u(t-\tau)(1-\lambda)+K_{1} v_{2}(t-\tau)\right]} \\
& -C_{2} D v_{1} \frac{v_{2}^{c}}{v_{2}}-C_{2} d_{2} v_{2}+C_{2} d_{2} v_{2}^{c} \\
& =\frac{\left(u-u^{c}\right)^{2}}{u}\left[R-\frac{R}{K}\left(u+u^{c}\right)\right]+\frac{A u^{c} v_{2}^{c}}{u}\left[\frac{u}{u^{c}}-\frac{u^{c}\left[u(1-\lambda)+K_{1} v_{2}\right]}{u\left[u^{c}(1-\lambda)+K_{1} v_{2}^{c}\right]}\right]-\frac{A K_{1} v_{2}^{c}\left[v_{2}^{c} u^{2}-v_{2} u^{c^{2}}\right]}{u^{2}\left[u^{c}(1-\lambda)+K_{1} v_{2}^{c}\right]} \\
& -A u v_{2}\left[\frac{\left[u^{c}(1-\lambda)+K_{1} v_{2}^{c}\right]}{u^{c}\left[u(1-\lambda)+K_{1} v_{2}\right]}-\frac{u^{c}}{u^{2}}\right]+\frac{A v_{2}}{u} \frac{K_{1}\left[v_{2}^{c} u^{2}-v_{2} u^{c^{2}}\right]}{u^{c}\left[u(1-\lambda)+K_{1} v_{2}\right]}+C_{1}\left(D+d_{1}\right) v_{1}^{c} \\
& +\frac{C_{1} B A(1-\lambda) u(t-\tau) v_{2}(t-\tau)}{u(t-\tau)(1-\lambda)+K_{1} v_{2}(t-\tau)} \\
& -\frac{C_{1} v_{1}^{c} B A(1-\lambda) u(t-\tau) v_{2}(t-\tau)}{v_{1}\left[u(t-\tau)(1-\lambda)+K_{1} v_{2}(t-\tau)\right]}-C_{2} D v_{1} \frac{v_{2}^{c}}{v_{2}}-C_{2} d_{2} v_{2}+C_{2} d_{2} v_{2}^{c}
\end{aligned}
$$

Define $V_{1}$ as

$$
V_{1}=V+C_{1} B A(1-\lambda) \int_{t-\tau}^{t}\left[\frac{u(s) v_{2}(s)}{\left[u(s)(1-\lambda)+K_{1} v_{2}(s)\right]}-\frac{u^{c} v_{2}^{c}}{\left[u^{c}(1-\lambda)+K_{1} v_{2}^{c}\right]}\right.
$$

$\left.-\frac{u^{c} v_{2}^{c}}{\left[u^{c}(1-\lambda)+K_{1} v_{2}^{c}\right]} \times \log \frac{\left[u^{c}(1-\lambda)+K_{1} v_{2}^{c}\right] u(s) v_{2}(s)}{u^{c} v_{2}^{c}\left[u(s)(1-\lambda)+K_{1} v_{2}(s)\right]}\right] d s$

From the above we get, 


$$
\begin{gathered}
\dot{V}_{1}=\frac{\left(u-u^{c}\right)^{2}}{u}\left[R-\frac{R}{K}\left(u+u^{c}\right)\right]+v_{2}^{c} A\left[1-\frac{u^{c^{2}}\left[u(1-\lambda)+K_{1} v_{2}\right]}{u^{2}\left[u^{c}(1-\lambda)+K_{1} v_{2}^{c}\right]}\right]+\frac{K_{1} A\left[v_{2}^{c} u^{2}-v_{2} u^{c^{2}}\right]}{u} \\
{\left[\frac{v_{2} u\left[u^{c}(1-\lambda)+K_{1} v_{2}^{c}\right]-v_{2}^{c} u^{c}\left[u(1-\lambda)+K_{1} v_{2}\right]}{u u^{c}\left[u(1-\lambda)+K_{1} v_{2}\right]\left[u^{c}(1-\lambda)+K_{1} v_{2}^{c}\right]}\right]+\frac{A v_{2} u^{c}}{u}+v_{2}^{c} A} \\
-\frac{v_{2}^{c} A v_{1}^{c}}{u^{c} v_{2}^{c} v_{1}}\left[\frac{\left[u^{c}(1-\lambda)+K_{1} v_{2}^{c}\right] u(t-\tau) v_{2}(t-\tau)}{\left[u(t-\tau)(1-\lambda)+K_{1} v_{2}(t-\tau)\right]}\right]-v_{2}^{c} A \frac{v_{1} v_{2}^{c}}{v_{1}^{c} v_{2}}
\end{gathered}
$$

$-A v_{2}+v_{2}^{c} A+v_{2}^{c} A \log \frac{\left[u^{c}(1-\lambda)+K_{1} v_{2}^{c}\right] u(t-\tau) v_{2}(t-\tau)}{u^{c} v_{2}^{c}\left[u(t-\tau)(1-\lambda)+K_{1} v_{2}(t-\tau)\right]}+v_{2}^{c} A \log \frac{u^{c} v_{2}^{c}\left[u(1-\lambda)+K_{1} v_{2}\right]}{\left[u^{c}(1-\lambda)+K_{1} v_{2}^{c}\right] u v_{2}}$

Note that

and

$$
C_{1}\left(D+d_{1}\right) v_{1}^{c}=C_{2} d_{2} v_{2}^{c}=v_{2}^{c} A=\frac{C_{1} B A(1-\lambda) u^{c} v_{2}^{c}}{u^{c}(1-\lambda)+K_{1} v_{2}^{c}}
$$

$$
\begin{gathered}
{\left[1-\frac{u^{c}}{u}\right] \frac{A u^{c}(1-\lambda) v_{2}^{c}}{\left[u^{c}(1-\lambda)+K_{1} v_{2}^{c}\right]}=\frac{A u^{c} v_{2}^{c}}{u}\left[\frac{u}{u^{c}}-\frac{u^{c}\left[u(1-\lambda)+K_{1} v\right]}{u\left[u^{c}(1-\lambda)+K_{1} v_{2}^{c}\right]}\right]-\frac{A v_{2} K_{1}}{u\left[u^{c}(1-\lambda)+K_{1} v_{2}^{c}\right]}\left[\frac{v_{2}^{c} u^{2}-v_{2} u^{c^{2}}}{u}\right]} \\
\dot{V_{1}}=\frac{\left(u-u^{c}\right)^{2}}{u}\left[R-\frac{R}{K}\left(u+u^{c}\right)\right]+\frac{K_{1} A\left[v_{2}^{c} u^{2}-v_{2} u^{c^{2}}\right]}{u}\left[\frac{v_{2} u\left[u^{c}(1-\lambda)+K_{1} v_{2}^{c}\right]-v_{2}^{c} u^{c}\left[u(1-\lambda)+K_{1} v_{2}\right]}{u u^{c}\left[u(1-\lambda)+K_{1} v_{2}\right]\left[u^{c}(1-\lambda)+K_{1} v_{2}^{c}\right]}\right] \\
-v_{2}^{c} A\left[\frac{u^{c^{2}}\left[u(1-\lambda)+K_{1} v_{2}\right]}{u^{2}\left[u^{c}(1-\lambda)+K_{1} v_{2}^{c}\right]}-1-\log \frac{u^{c^{2}}\left[u(1-\lambda)+K_{1} v_{2}\right]}{u^{2}\left[u^{c}(1-\lambda)+K_{1} v_{2}^{c}\right]}\right] \\
-v_{2}^{c} A\left[\frac{v_{1}^{c}}{u^{c} v_{2}^{c} v_{1}} \frac{\left[u^{c}(1-\lambda)+K_{1} v_{2}^{c}\right] u(t-\tau) v_{2}(t-\tau)}{\left[u(t-\tau)(1-\lambda)+K_{1} v_{2}(t-\tau)\right]}-1\right. \\
\left.-\log \frac{v_{1}^{c}}{u^{c} v_{2}^{c} v_{1}} \frac{\left[u^{c}(1-\lambda)+K_{1} v_{2}^{c}\right] u(t-\tau) v_{2}(t-\tau)}{\left[u(t-\tau)(1-\lambda)+K_{1} v_{2}(t-\tau)\right]}\right] \\
\left.\dot{V}_{1} \leq-\frac{v_{2}^{c} A\left[\frac{v_{1} v_{2}^{c}}{v_{1}^{c} v_{2}}-1-\log \frac{v_{1} v_{2}^{c}}{v_{1}^{c} v_{2}}\right]-v_{2}^{c} A \log \frac{u^{c}}{u}-A v_{2}\left(u-\frac{u^{c}}{u}\right)}{u}-u^{c}\right)^{2}\left[\frac{R}{K}\left(u+u^{c}\right)-R-\rho_{1}\right]-\frac{K_{1} A\left(v_{2}-v_{2}^{c}\right)^{2}\left[u^{c^{2}}(1-\lambda)-\rho_{2}\right]}{u\left[u(1-\lambda)+K_{1} v_{2}\right]\left[u^{c}(1-\lambda)+K_{1} v_{2}^{c}\right]} \\
-v_{2}^{c} A\left[\frac{u^{c^{2}}\left[u(1-\lambda)+K_{1} v_{2}\right]}{u^{2}\left[u^{c}(1-\lambda)+K_{1} v_{2}^{c}\right]}-1-\log \frac{u^{c^{2}}\left[u(1-\lambda)+K_{1} v_{2}\right]}{u^{2}\left[u^{c}(1-\lambda)+K_{1} v_{2}^{c}\right]}\right] \\
-v_{2}^{c} A\left[\frac{v_{1}^{c}}{u^{c} v_{2}^{c} v_{1}} \frac{\left[u^{c}(1-\lambda)+K_{1} v_{2}^{c}\right] u(t-\tau) v_{2}(t-\tau)}{\left[u(t-\tau)(1-\lambda)+K_{1} v_{2}(t-\tau)\right]}-1\right. \\
\left.-\log \frac{v_{1}^{c}}{u^{c} v_{2}^{c} v_{1}} \frac{\left[u^{c}(1-\lambda)+K_{1} v_{2}^{c}\right] u(t-\tau) v_{2}(t-\tau)}{\left[u(t-\tau)(1-\lambda)+K_{1} v_{2}(t-\tau)\right]}\right]
\end{gathered}
$$

$-v_{2}^{c} A\left[\frac{v_{1} v_{2}^{c}}{v_{1}^{c} v_{2}}-1-\log \frac{v_{1} v_{2}^{c}}{v_{1}^{c} v_{2}}\right]-v_{2}^{c} A \log \frac{u^{c}}{u}-A v_{2}\left(u-\frac{u^{c}}{u}\right)$

where

$$
\rho_{1}=\frac{A K_{1} u^{2} u^{c} v_{2}^{c}(1-\lambda)+u^{c^{2}} v_{2} K_{1} v_{2}^{c}+2 u v_{2}^{c^{2}} K_{1} v_{2}}{2 u u^{c}\left[u(1-\lambda)+K_{1} v_{2}\right]\left[u^{c}(1-\lambda)+K_{1} v_{2}^{c}\right]}
$$




$$
\begin{gathered}
\text { Delay and Prey Taking Refug } \\
\rho_{2}=\frac{v_{2}^{c}\left[u^{2}(1-\lambda)+u^{c} K_{1} v_{2}^{c}\right]}{2 u}
\end{gathered}
$$

Hence if (35) holds, then it follows from (41) that $\dot{V}(t) \leq 0$, with equality if and only if $u=u^{c}$, $\frac{v_{1}^{c}\left[u^{c}(1-\lambda)+K_{1} v_{2}^{c}\right] u(t-\tau) v_{2}(t-\tau)}{u^{c} v_{2}^{c} v_{1}\left[u(t-\tau)(1-\lambda)+K_{1} v_{2}(t-\tau)\right]}=\frac{v_{1} v_{2}^{c}}{v_{1}^{c} v_{2}}=1$.Looking for the invariant $\mathcal{H}$ within the set $H=\left\{\left(u, v_{1}, v_{2}\right) ; u=\right.$ $\left.u^{c}, \frac{v_{1}^{c}\left[u^{c}(1-\lambda)+K_{1} v_{2}^{c}\right] u(t-\tau) v_{2}(t-\tau)}{u^{c} v_{2}^{c} v_{1}\left[u(t-\tau)(1-\lambda)+K_{1} v_{2}(t-\tau)\right]}=\frac{v_{1} v_{2}^{c}}{v_{1}^{c} v_{2}}=1\right\}$. Here $u=u^{c} \quad$ on $\quad \mathcal{H} \quad$ and $\quad$ also $0=\dot{u}(t)=R u^{c}\left(1-\frac{u^{c}}{K}\right)-$ $\frac{A u^{c}(1-\lambda) v_{2}}{\left.u^{c}(1-\lambda)+K_{1} v_{2}\right)}$, hence we get $v_{2}(t)=v_{2}^{c}$. From (3) $0=\dot{v}_{2}(t)=D v_{1}(t)-d_{2} v_{2}^{c}$ which gives $v_{1}=v_{1}^{c}$. Therefore, the only invariant set in $\mathrm{H}$ is $\mathcal{H}=\left\{\left(u^{c}, v_{1}^{c}, v_{2}^{c}\right)\right\}$. Using Lasalle invariance principle $E_{c}$ is globally attractive for the system.

\section{Numerical Simulations}

Example 5.1 Consider the system (1-3) with $\lambda=.5, \tau=6, \mathrm{R}=.65, \mathrm{D}=.9, \mathrm{~d}_{1}=.9, \mathrm{~d}_{2}=.6, \mathrm{~B}=.6, \mathrm{~A}=.9, \mathrm{~K}_{1}=$ $.9, \mathrm{~K}=7$. Calculations shows $B A-r_{1}=-0.66<0$ and predator free equilibrium $E_{1}=(7,0,0)$. By theorem (2.3) $E_{1}$ is locally asymptotically stable. Figure 1 and Figure 2.

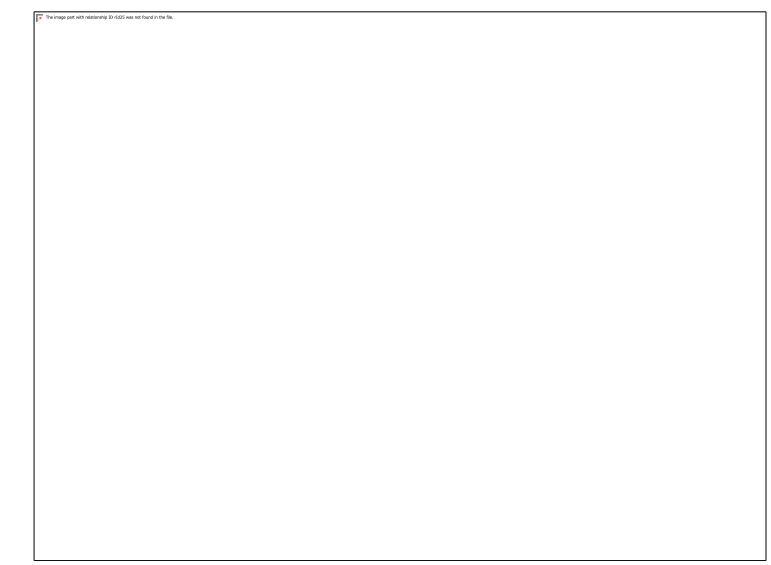

Figure1. The boundary equilibrium $E_{1}=(7,0,0)$ is locally asymptotically stable

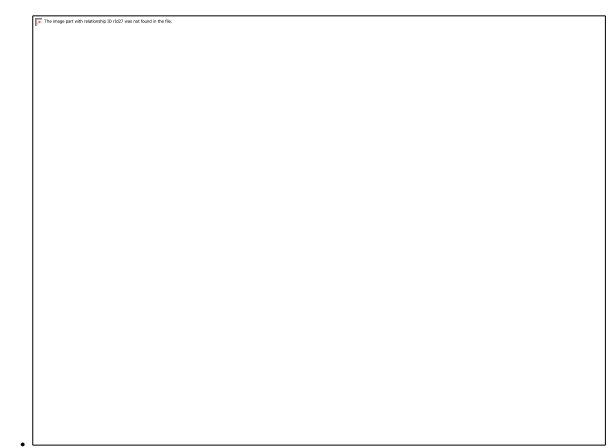

Figure 2. The boundary equilibrium $E_{1}=(7,0,0)$ at different initial values is locally asymptotically stable

Example 5.2 For the system (1-3) when $\lambda=.3, \mathrm{R}=4, \mathrm{D}=3, \mathrm{~d}_{1}=1, \mathrm{~d}_{2}=1, \mathrm{~B}=3, \mathrm{~A}=2, \mathrm{~K}_{1}=3, \mathrm{~K}=10$ and $\tau$ $=5$, we find $B A-r_{1}=4.6667>0,36=K_{1} R B>(1-\lambda)\left(B A-r_{1}\right)=3.2667$. Also $P_{1}=7.5051, P_{2}=$ $7.5564, P_{4}=38.2518$ and $E_{c}=(9.0926,2.4752,7.4256)$. The conditions in theorem (2.4)(1) are satisfied and hence $E_{c}$ is locally asymptotically stable. Figure 3 and Figure 4.

Figure 3. The coexistence equilibrium $E_{\mathrm{c}}=(9.0926,2.4752,7.4256)$ is locally asymptotically stable 
Figure 4. The coexistence equilibrium $E_{\mathrm{c}}=(9.0926,2.4752,7.4256)$ at different initial values is locally asymptotically stable

Example 5.3 Let for the system $(1-3) \lambda=.4, \mathrm{R}=.8, \mathrm{D}=.9, \mathrm{~d}_{1}=.3, \mathrm{~d}_{2}=.1, \mathrm{~B}=.6, \mathrm{~A}=.7, \mathrm{~K}_{1}=.5, \mathrm{~K}=20$ and $\tau=5$. Calculation yields $B A-r_{1}=0.2867>0,0.24=K_{1} R B>(1-\lambda)\left(B A-r_{1}\right)=0.172 . P_{1}=$ $0.7504, P_{2}=0.8447, P_{4}=1.8730$ and $E_{c}=(5.6667,1.6244,14.62)$. The conditions in theorem $(2.4)(2)$ are met. Also $\tau_{0}=14.3451$. When $\tau=12<14.3451=\tau_{0}$, the equilibrium $E_{c}$ is locally asymptotically stable. Figure 5 and Figure 6. When $\tau=16>14.3451=\tau_{0}$, the equilibrium $E_{c}$ yields a periodic solution. Figure 7 and Figure 8 .

Figure 5. The coexistence equilibrium $E_{\mathrm{c}}=(5.6667,1.6244,14.62)$ is locally asymptotically stable for $\tau=12$

Figure 6. The coexistence equilibrium $E_{\mathrm{c}}=(5.6667,1.6244,14.62)$ is locally asymptotically stable for $\tau=12<14.3451=\tau_{0}$ as time increases.

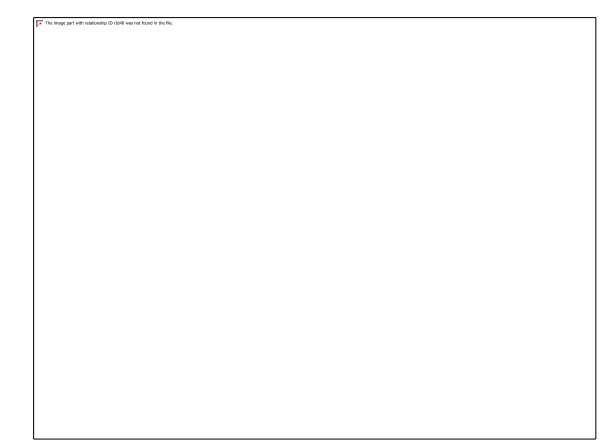

Figure 7. The coexistence equilibrium $E_{\mathrm{c}}=(5.6667,1.6244,14.62)$ undergoes oscillation 
for $\tau=16>14.3451=\tau_{0}$

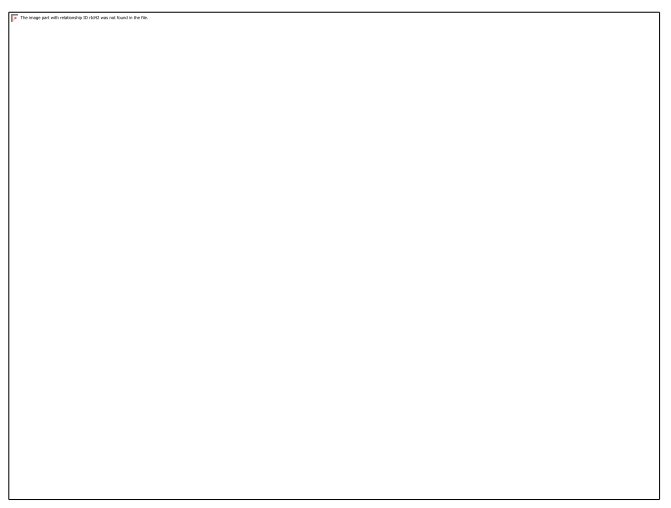

Figure 8. The coexistence equilibrium $E_{\mathrm{c}}=(5.6667,1.6244,14.62)$ undergoes oscillation for

$$
\tau=16>14.3451=\tau_{0}
$$

Example 5.4 In (1) let $\lambda=0.7, \mathrm{R}=4, \mathrm{D}=3, \mathrm{~d}_{1}=1, \mathrm{~d}_{2}=1, \mathrm{~B}=3, \mathrm{~A}=2, \mathrm{~K}_{1}=3, \mathrm{~K}=20$ and $\tau=5$. Calculation shows that $B A-r_{1}=4.66>0,4=R>\frac{A(1-\lambda)}{K_{1}}=0.2,3.64=\frac{R}{K}\left(l_{1}+u^{c}\right)-R>\overline{\rho_{1}}=0.474$ and $110=$ $u^{c^{2}}(1-\lambda) \geq \overline{\rho_{2}}=89.909$.hence by theorem (4.1), the positive equilibrium $E_{c}=(19.2222,2.2425,6.723)$ is stable globally. Figure 9.

Figure 9. The coexistence equilibrium $E_{\mathrm{c}}=(19.222,2.2425,6.723)$ with different initial values is globally stable

\section{Conclusion}

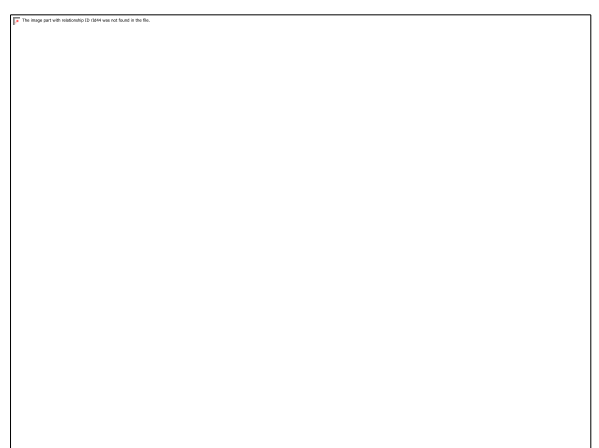

The mathematical model of the prey predator system considered for analysis is uniformly bounded which implies that the model is well behaved biologically. The boundary equilibrium is asymptotically stable under appropriate conditions. The conditions for the coexistence equilibrium to be locally stable is obtained. Also, it is found that the delay in time can make the stable equilibrium to be unstable, as the time lag crosses a critical value and making way for Hopf bifurcation. By suitable construction of the Lyapunov functional global stability at the positive equilibrium point is established.

\section{Conflicts of Interest}

No conflict of interest was declared by the authors.

\section{References}

1. Kar, T. K., "Modelling and analysis of a harvested prey-predator system incorporating a prey refuge", Journal of Computational and Applied Mathematics, 185(1): 19-33, (2006).

2. Wang, L. and Feng, G., "Global Stability and Hopf Bifurcation of a Predator-Prey Model with Time Delay and Stage Structure", Journal of Applied Mathematics, (2014). https://doi.org/10.1155/2014/431671.

3. Wang, L. and Xu, R., "Global dynamics of a predator-prey model with stage structure and delayed predator response", Discrete Dynamics in Nature and Society, (2013).

4. https://doi.org/10.1155/2013/724325.

5. Yang, W.S., Li, X. P. and Bai, Z. J., "Permanence of periodic Holling type-IV predator-prey system with stage structure for prey", Mathematical and Computer Modelling, 48(5-6):677-684, (2008).

6. Liang, Z. and Pan, H., "Qualitative analysis of a ratio-dependent Holling-Tanner model”, Journal of Mathematical Analysis and Applications, 334(2): 954-964, (2007).

7. Saha, T. and Chakrabarti, C., "Dynamical analysis of a delayed ratio-dependent Holling-Tanner predator-prey model", Journal of Mathematical Analysis and Applications, 358(2): 389-402, (2009).

8. Flores, J. D. and González-Olivares, E., "A predator-prey model with ratio-dependent functional response and strong Allee effect on prey", Recent Advances in Fluid Mechanics, Heat \& Mass Transfer and Biology: 127-132, (2012). 
9. Ashine, A. B. and Gebru, D. M., "Mathematical modeling of a predator-prey model with modified Leslie-Gower and Holling type-II schemes", Mathematics and Decision Sciences, 17: 20-40, (2017).

10. Abid, W., Yafia, R., Aziz-Alaoui, M. A and Ahmed Aghriche., "Dynamics analysis and optimality in selective harvesting predator-prey model with modified Leslie-Gower and Holling-type II", Nonautonomous Dynamical Systems, 6: 1-17, (2019).

11. Abdulghafour, A. S. and Naji, R. K., "The impact of refuge and harvesting on the dynamics of preypredator system", Science International (Lahore), 30(2): 315-323, (2018).

12. Xu, R. and Ma. Z., "Stability and Hopf bifurcation in a ratio-dependent predator-prey system with stage structure", Chaos, Solitons and Fractals, 38: 669-684, (2008).

13. Yu, X., Zhu, Z., Lai, L. and Chen, F., "Stability and bifurcation analysis in a single-species stage structure system with Michaelis-Menten-type harvesting”, Advances in Difference Equations, (2020). https://doi.org/10.1186/s13662-020-02652-7.

14. Dai, Y., Lin, Y. and Zhao, H.,"Hopf bifurcation and global periodic solutions in a predator-prey system with Michealis-Menten type functional response and two delays", Abstract and Applied Analysis, (2014). https://doi.org/10.1155/2014/835310.

15. Naji, R. K. and Majeed, S. J., "The dynamical analysis of a prey-predator model with a refuge-stage structure prey population", International Journal of Differential Equations, (2016). https://doi.org/10.1155/2016/2010464.

16. Kant, S. and Kumar, V., "Dynamical behavior of a stage structured prey-predator model", International Journal of Nonlinear Analysisand Applications, 7(1): 231-241, (2016).

17. Li, B., Liu, S., Cui, J. and Li, J., "A simple predator prey population model with rich dynamics", Applied Sciences, (2016).https://doi.org/10.3390/app6050151.

18. Mortoja, S. G., Panja, P. and Mondal, S. K., "Dynamics of a predator-prey model with stage-structure on both species and anti-predator behavior", Informatics in Medicine Unlocked, 10: 50-57, (2018).

19. Lajmiri, Z., Ghaziani, R. K. and Orak, I., "Bifurcation and stability analysis of a ratio-dependent predator-prey model with predator harvesting rate", Chaos, Solitons and Fractals, 106: 193-200, (2018).

20. Jost, C., Arino, O. and Arditi, R., "About deterministic extinction in ratio-dependent predator-prey model”, Bulletin of Mathematical Biology, 61(1): 19-32, (1999).

21. Sambath, M. and Balachandran, K., "Spatiotemporal dynamics of a predator-prey model incorporating a prey refuge", Journal of Applied Analysis and Computation, 3(1): 71-80, (2013).

22.

23. Xiao, Z., Li, Z., Zhu, Z. and Chen, F., "Hopf bifurcation and stability in a Beddington-DeAngelis predator-prey model with stage structure for predator and time delay incorporating prey refuge", Open Mathematics, 17(1): 141-159, (2019).

24. Kuang, Y., Delay Differential Equations:With Applications in Population Dynamics. Academic Press, Newyork, (1993).

25. Chen F.D., Li Z. and Huang Y. J., "Note on the Permanence of the competitive system with infinite delay and feedback controls”, Nonlinear Analysis:Real World Applications, 8(2): 680-687, (2007). 